\title{
Harmony in Electrons: Cyclotron and Synchrotron Emission by Thermal Electrons in a Magnetic Field
}

\section{Citation}

Mahadevan, Rohan, Ramesh Narayan, and Insu Yi. 1996. "Harmony in Electrons: Cyclotron and Synchrotron Emission by Thermal Electrons in a Magnetic Field." The Astrophysical Journal 465 (July): 327. https://doi.org/10.1086/177422.

\section{Permanent link}

http://nrs.harvard.edu/urn-3:HUL.InstRepos:41384903

\section{Terms of Use}

This article was downloaded from Harvard University's DASH repository, and is made available under the terms and conditions applicable to Other Posted Material, as set forth at http:// nrs.harvard.edu/urn-3:HUL.InstRepos:dash.current.terms-of-use\#LAA

\section{Share Your Story}

The Harvard community has made this article openly available.

Please share how this access benefits you. Submit a story.

Accessibility 


\title{
Harmony in Electrons: Cyclotron and Synchrotron Emission by Thermal Electrons in a Magnetic Field.
}

\author{
Rohan Mahadevan, Ramesh Narayan, and Insu Yi 1 \\ Harvard-Smithsonian Center for Astrophysics, 60 Garden St., Cambridge, MA 02138.
}

\begin{abstract}
We present a complete solution to the cyclotron-synchrotron radiation due to an isotropic distribution of electrons moving in a magnetic field. We make no approximations in the calculations other than artificially broadening the harmonics by a small amount in order to facilitate the numerics. In contrast to previous calculations, we sum over all relevant harmonics and integrate over all particle and observer angles relative to the magnetic field. We present emission spectra for electron temperatures $T=5 \times 10^{8} \mathrm{~K}, 10^{9} \mathrm{~K}, 2 \times 10^{9} \mathrm{~K}$ to $3.2 \times 10^{10} \mathrm{~K}$, and provide simple fitting formulae which give a fairly accurate representation of the detailed results. For $T \geq 3.2 \times 10^{10} \mathrm{~K}$, the spectrum is represented well by the asymptotic synchrotron formula, which is obtained by assuming that the radiating electrons have Lorentz factors large compared to unity. We give an improved fitting formula also for this asymptotic case.
\end{abstract}

\section{Introduction}

Radiation by quasi-relativistic and fully relativistic electrons in magnetic fields is very common in astrophysics. Examples of sources include supernova remnants (e.g. Anderson, Keohane \& Rudnick 1995), jets and lobes in radio galaxies (e.g. Carilli et al. 1991), hot accretion flows onto neutron stars and black holes (e.g. Narayan, Yi \& Mahadevan 1995), and relativistic fireballs in gamma-ray bursts (e.g. Paczyński \& Rhoads 1993, Meszaros, Laguna \& Rees 1993). In all these examples we have thermal or non-thermal electrons radiating cyclo-synchrotron radiation in a magnetic field which is probably of near-equipartition strength. It is clearly important to be able to calculate the radiative luminosities and spectra of these systems.

Surprisingly, a full solution to this problem does not appear to have been published so far, especially for thermal sources. The majority of previous work (e.g. Schott 1912, Schwinger 1949, Oster 1960, 1961, Pacholczyk 1970) is limited either to the cyclotron

\footnotetext{
${ }^{1}$ Institute for Advanced Study, Princeton, NJ 08540.
} 
or the synchrotron limit where analytical formulae may be used. These calculations are not relevant in the transition zone between cyclotron and synchrotron radiation when the velocities of the electrons are quasirelativistic. Petrosian (1981) has obtained some approximate formulae in this intermediate regime, but a complete analysis of the emission from thermal sources with temperatures in the range $10^{9}-10^{10} \mathrm{~K}$ can only be done numerically. Takahara \& Tsuruta (1982) and Melia (1994) have reported numerical calculations in the mildly relativistic regime, but both calculations involve some approximations and do not agree with each other. Therefore, there is need for a more accurate treatment to resolve this difference. In particular, we find significant differences between the results quoted by Melia (1994) and the exact calculations presented here.

We present in this paper the complete solution to the cyclo-synchrotron problem for a thermal plasma. In $\S 2$. we give a qualitative as well as quantitative description of the problem, and briefly discuss previous work in this subject. We also present an outline of how we solve the problem exactly. Then in $\$ 3$. we present the results. We calculate the emission due to a thermal distribution of electrons for a range of temperatures, and provide analytic fitting functions for the spectra.

\section{Theory}

\subsection{Qualitative Features}

A non-relativistic particle moving in a magnetic field radiates with a simple dipole pattern (e.g. Rybicki \& Lightman 1979) so that an observer sees a sinusoidal electric field. The observed spectrum consists of a single delta function at the orbital frequency, $\omega_{o}$, of the electron. This is the limit of extreme cyclotron radiation. If the particle is speeded up, the dipole radiation pattern gets distorted and the observed electric field is no longer purely sinusoidal. The spectrum then picks up additional Fourier components, or harmonics, which are integral multiples of the fundamental frequency. The situation is illustrated in Fig. 1a. At the same time, $\omega_{o}$ decreases with increasing velocity $v$, according to

$$
\omega_{o}=\frac{\omega_{b}}{\gamma}, \quad \omega_{b}=\frac{e B}{m_{e} c},
$$

where $\omega_{b}$ is the cyclotron frequency of the particle, and $\gamma=1 / \sqrt{1-v^{2} / c^{2}}=1 / \sqrt{1-\beta^{2}}$ is the Lorentz factor.

With increasing $\gamma$, the emission is beamed more and more towards the direction of motion of the particle. For large $\gamma$, the electric field appears as a series of delta function-like pulses, each of width $\sim 1 / \gamma^{2} \omega_{b}$, with succeeding pulses separated by a 
time $2 \pi \gamma / \omega_{b}$. The Fourier transform of these delta functions gives closely spaced delta functions in frequency which approximate a nearly continuous spectrum extending up to $\omega \sim \gamma^{2} \omega_{b}$, and cutting off exponentially at higher $\omega$. This corresponds to the synchrotron limit, where the spectrum has the universal shape shown in Fig. 1b.

The cyclotron and synchrotron limits have very different spectra, as Fig. 1 shows. How does the spectrum evolve from one to the other as the particle velocity is increased? Answering this question requires detailed calculations and is the topic of this paper.

\subsection{Previous Work}

We begin by reviewing a number of well-known results. The power (energy/time/steradian/frequency) emitted by an electron moving with a velocity parameter between $\vec{\beta}$ and $\vec{\beta}+d \vec{\beta}$, in a frequency range $d \omega$, and at an observer angle $\theta$ with respect to the magnetic field, is given by (e.g. Schott 1912, Rosner 1958, Bekefi 1966)

$$
\eta_{\omega}(\vec{\beta}, \theta) d \omega=\frac{e^{2} \omega^{2}}{2 \pi c}\left[\sum_{m=1}^{\infty}\left(\frac{\cos \theta-\beta_{\|}}{\sin \theta}\right)^{2} J_{m}^{2}(x)+\beta_{\perp}^{2} J_{m}^{\prime 2}(x)\right] \delta(y) d \omega
$$

Here

$$
\begin{gathered}
x=\frac{\omega}{\omega_{o}} \beta_{\perp} \sin \theta, \\
y=m \omega_{o}-\omega\left(1-\beta_{\|} \cos \theta\right),
\end{gathered}
$$

$\delta(y)$ is the Dirac delta function, $J_{m}(x)$ is the Bessel function of order $m, J_{m}^{\prime}(x)$ is its derivative, $\beta_{\|}=\beta \cos \theta_{p}$ is the velocity parameter parallel to the magnetic field $B$, where the particle moves at an angle $\theta_{p}$ to the local field, and $\beta_{\perp}=\beta \sin \theta_{p}$ is the velocity parameter perpendicular to the magnetic field.

Each integer $m$ in the summation in equation (2) corresponds to a harmonic, and the presence of the $\delta$-function implies that the emission occurs at discrete frequencies. To calculate the power in successive harmonics at low $\beta$ (the cyclotron limit), we expand the Bessel functions to lowest order in $x$. After integrating over all observer angles, the emission in each harmonic is given by (Schwinger 1949, Rosner 1956, Bekefi 1966)

$$
\eta_{m}^{T}=\frac{2 e^{2} \omega_{b}^{2}}{c} \frac{(m+1)\left(m^{2 m+1}\right)}{(2 m+1) !} \beta^{2 m},
$$

This result, which is valid so long as $m \beta \ll 1$, shows that the spectrum decreases rapidly with increasing $m$. Fig. 1a shows an example with $\beta=0.1$, which corresponds to this limit. 
In the opposite limit of $\gamma \gg 1$, which is the synchrotron limit, we are interested in large $m$ 's, and the Bessel functions can be approximated by modified Bessel functions (Bekefi 1966). We then have the familiar result (e.g. Schwinger 1949, Pacholczyk 1970, Rybicki \& Lightman 1979)

$$
\frac{d E}{d \omega}=\frac{\sqrt{3} e^{3} B \sin \theta_{p}}{2 \pi m_{e} c^{2}} F(X)
$$

where

$$
X=\frac{\omega}{\omega_{c}}, \quad \omega_{c}=\frac{3}{2} \gamma^{2} \omega_{b} \sin \theta_{p}
$$

$F(X)$ is given by

$$
F(X) \equiv X \int_{X}^{\infty} K_{\frac{5}{3}}(\xi) d \xi
$$

and $K_{\frac{5}{3}}(\xi)$ is the modified Bessel function. By taking appropriate limits of $F(X)$, we obtain

$$
\begin{aligned}
& F(X) \rightarrow \frac{4 \pi}{\sqrt{3} \Gamma\left(\frac{1}{3}\right)}\left(\frac{X}{2}\right)^{1 / 3}, \quad X \ll 1, \\
& F(X) \rightarrow\left(\frac{\pi}{2}\right)^{1 / 2} e^{-X} X^{1 / 2}, \quad X \gg 1 .
\end{aligned}
$$

Fig. 1b shows the synchrotron spectrum for a particle with $\gamma=10$.

The above results are for electrons of a given velocity. For a thermal distribution we need to integrate the emission over a Maxwellian. This integral can be done in the synchrotron limit to give (Pacholczyk 1970),

$$
\varepsilon_{\omega} d \omega=C \frac{\chi}{K_{2}\left(1 / \theta_{e}\right)} I\left(\frac{x_{M}}{\sin \theta_{p}}\right) d \omega \operatorname{ergs~s}^{-1} \mathrm{~Hz}^{-1},
$$

where

$$
C \equiv \frac{e^{2} n_{e} \omega_{b}}{\sqrt{3} \pi c}, \quad \chi \equiv \frac{\omega}{\omega_{b}}, \quad x_{M} \equiv \frac{2 \chi}{3 \theta_{e}^{2}}, \quad \theta_{e} \equiv \frac{k T}{m_{e} c^{2}},
$$

$K_{2}(x)$ is the modified Bessel function, and $I\left(x_{M}\right)$ is defined by

$$
I\left(x_{M}\right) \equiv \frac{1}{x_{M}} \int_{0}^{\infty} z^{2} \exp (-z) F\left(x_{M} / z^{2}\right) d z .
$$

In writing equation (11), we have included the proper normalization for a relativistic Maxwellian distribution; in the limit of high temperatures, this normalization is equivalent to that used by Pacholczyk (1970). The limiting behavior of $I\left(x_{M}\right)$ for small and large $x_{M}$ are straightforward. The former has been worked out by Pacholczyk (see Eq. (A2)), and the latter by Petrosian (1981) (see Eq. (A4). Equation (11) corresponds to particles at a fixed angle $\theta_{p}$ to the magnetic field. For an isotropic 
particle distribution, we need to perform another integral over $d\left(\cos \theta_{p}\right)$. The asymptotic dependencies of the result for small and large $x_{M}$ are again easy to calculate and are discussed in Appendix A.

For the mildly relativistic case, Petrosian (1981) has obtained the following analytic approximations for the emission spectrum from a thermal distribution of electrons as a function of the observer angle $\theta$ :

$$
\varepsilon_{\omega}(\theta) d \omega \rightarrow \frac{2 \pi}{6^{1 / 2}} C \frac{\chi}{K_{2}\left(1 / \theta_{e}\right)} \exp \left(-6.75^{1 / 3} x_{M}^{1 / 3}\right) d \omega, \quad \chi \theta_{e} \gg 1
$$

and

$$
\begin{aligned}
\varepsilon_{\omega}(\theta) d \omega & \rightarrow 3^{1 / 2} 2^{3 / 2} \pi^{2} C \frac{1}{\theta_{e} K_{2}\left(1 / \theta_{e}\right)}\left(\chi \theta_{e}\right)^{3 / 2} \\
& \times\left(\frac{1+\cos ^{2} \theta}{\sin ^{2} \theta}\right) \exp \left[-\chi \ln \left(\frac{2}{e \chi \theta_{e} \sin ^{2} \theta}\right)\right] d \omega, \quad \theta_{e} \ll 1 .
\end{aligned}
$$

These results are valid only with the additional condition $\omega / \omega_{b} \gg 1$, the regime Petrosian (1981) was interested in. For $x_{M} \gg 1$, equation (14) is exactly the same as equation (11) (cf. Eq. A4), and we see that the approximations made by Petrosian (1981) in this regime are the same as the extreme synchrotron approximations.

The extreme synchrotron results described above provide quite an accurate representation of the exact results for electron temperatures $\gtrsim 3 \times 10^{10} \mathrm{~K}$, while the series result given in equation (5) is very good at temperatures $\lesssim 10^{8} \mathrm{~K}$. For $10^{8} \mathrm{~K}$ $\lesssim T \lesssim 3 \times 10^{10} \mathrm{~K}$, we cannot use either of these limiting results but must solve the complete cyclo-synchrotron problem.

\subsection{The Complete Solution}

We start by writing the integrals that need to be carried out. To determine the luminosity, $L_{\omega} \equiv d E / d \omega$, due to a particle moving with velocity parameter $\beta$, we must integrate $\eta_{\omega}(\vec{\beta}, \theta)$ (Eq. (国)) over observer angles, and a given particle distribution:

$$
L_{\omega} \equiv \frac{d E}{d \omega}=\frac{2}{4 \pi} \int_{0}^{1} d \beta n(\beta) \int_{0}^{2 \pi} d \phi_{p} \int_{0}^{1} d\left(\cos \theta_{p}\right) \int_{0}^{2 \pi} d \phi \int_{-1}^{1} d(\cos \theta) \eta_{\omega}(\vec{\beta}, \theta),
$$

where $\eta_{\omega}(\vec{\beta}, \theta)$ is given by Eq. (2) $)$. The subscript $p$ refers to the particle, and $n(\beta)$ is the velocity distribution of the particles, which is taken to be isotropic. The factor of 2 in front is because we integrate over only half the range of $\cos \theta_{p}$, and the factor of $1 / 4 \pi$ 
comes from the angular normalization of the (isotropic) particle distribution function. The integrals over $\phi_{p}$ and $\phi$ are trivial, giving

$$
L_{\omega} \equiv \frac{d E}{d \omega}=2 \pi \int_{0}^{1} d \beta n(\beta) \int_{0}^{1} d\left(\cos \theta_{p}\right) \int_{-1}^{1} d(\cos \theta) \eta_{\omega}(\vec{\beta}, \theta) .
$$

For a fixed velocity parameter $\beta$ and frequency $\omega$, we numerically evaluate the two innermost integrals in Eq. (17), as well as the sum over harmonics in the expression (2) for $\eta_{\omega}(\vec{\beta}, \theta)$. We repeat this calculation for various values of $\beta$ and $\omega$ and tabulate the results. The results can then be convolved with any isotropic velocity distribution to obtain the spectrum $L_{\omega}$. In this paper, we restrict ourselves to a relativistic Maxwellian $n(\beta)$ and present detailed results for this particular case.

The evaluation of Eq. (17) involves a delta function (Eq. (2)), which determines the precise frequencies at which radiation is observed. For a given harmonic $m$, the delta function implies that there is emission only at

$$
\omega=\frac{m \omega_{o}}{\left(1-\beta_{\|} \cos \theta\right)}
$$

Since $m$ takes on only integer values, this means that for a given $\vec{\beta}$ and $\cos \theta$, emission is observed only at a discrete set of $\omega$. Unfortunately, the discrete nature of the emission poses a serious numerical difficulty since it requires infinite resolution in frequency. In order to make the numerics tractable, we replace the delta function with a smooth broadening function, $f(\omega)$, which is nonzero over a finite frequency range $\omega_{c} \pm \Delta \omega$. Here $\omega_{c}$ is the central frequency where we wish to evaluate the emission, and $\Delta \omega$ is a broadening width, which we adjust. With the smoothing function, the delta function in equation (2) becomes

$$
\delta(y)=\delta\left[m \omega_{o}-\omega\left(1-\beta_{\|} \cos \theta\right)\right]=\frac{1}{\omega_{b}} \frac{1}{1-\beta_{\|} \cos \theta} f(\chi),
$$

where

$$
\chi=\frac{\omega}{\omega_{b}}=\frac{\omega}{\gamma \omega_{o}} .
$$

For $f(\chi)$, we choose the functional form

$$
f(\chi)=\frac{15}{16 \Delta \chi}\left[1-\left(\frac{2}{\Delta \chi^{2}}\right)\left(\chi-\chi_{c}\right)^{2}+\left(\frac{1}{\Delta \chi^{4}}\right)\left(\chi-\chi_{c}\right)^{4}\right]
$$

which has the property that $f\left(\omega_{c} \pm \Delta \omega\right)=f^{\prime}\left(\omega_{c} \pm \Delta \omega\right)=0$.

In our calculations we set $\Delta \chi=\alpha \chi$, and we have found that a choice $\alpha=0.05$ allows the delta function to be broadened sufficiently to stabilize the numerics without losing too many details of the harmonic structure of the emission. For each value of $\chi_{c}$ of interest, we calculate the power emitted into a width $2 \Delta \chi$ centered at $\chi_{c}$, counting 
only those harmonics which fall within this width. With Eqs. (2), and (19), Eq. (17) thus becomes

$$
\begin{aligned}
& L_{\omega}=2 \pi\left(\frac{e^{2} \omega_{b}}{2 \pi c}\right) \int_{0}^{1} d \beta n(\beta) \int_{0}^{1} d\left(\cos \theta_{p}\right) \int_{-1}^{1} d(\cos \theta) \frac{f\left(\chi^{\prime}\right)}{1-\beta_{\|} \cos \theta} \\
& \times \quad \chi^{\prime 2}\left[\sum_{m=1}^{\infty}\left(\frac{\cos \theta-\beta_{\|}}{\sin \theta}\right)^{2} J_{m}^{2}\left(\gamma \chi^{\prime} \frac{\beta_{\perp} \sin \theta}{1-\beta_{\|} \cos \theta}\right)+\beta_{\perp}^{2} J_{m}^{\prime 2}\left(\gamma \chi^{\prime} \frac{\beta_{\perp} \sin \theta}{1-\beta_{\|} \cos \theta}\right)\right]
\end{aligned}
$$

For a Maxwellian velocity distribution with temperature $T$, we have

$$
n(\gamma) d \gamma=\frac{m_{e} c^{2}}{k T K_{2}\left(1 / \theta_{e}\right)} \beta \gamma^{2} \exp \left(-\gamma / \theta_{e}\right) d \gamma
$$

or

$$
n(\beta) d \beta=\frac{m_{e} c^{2}}{k T K_{2}\left(1 / \theta_{e}\right)} \beta^{2} \gamma^{5} \exp \left(-\gamma / \theta_{e}\right) d \beta
$$

with

$$
\int_{1}^{\infty} n(\gamma) d \gamma=\int_{0}^{1} n(\beta) d \beta=1
$$

Here $k$ is Boltzmann's constant, $\theta_{e}=k T / m_{e} c^{2}$, and $K_{2}(x)$ is the modified Bessel function.

The procedure to calculate the full cyclo-synchrotron emission spectrum is as follows. Using Eq. (22), we fix the values of $\beta, \cos \theta_{p}, \cos \theta$, and sum over the harmonics responsible for emission over the frequency range $\chi-\Delta \chi$ to $\chi+\Delta \chi$. This gives the total emission into the given observer angle $\theta$ from particles at angle $\theta_{p}$. We then integrate over all observer $(\cos \theta)$ directions, to get the emission into all of observer space, and integrate over all particle $\left(\cos \theta_{p}\right)$ directions to obtain the total emission per particle at the given value of $\chi$ due to an isotropic distribution of particles moving with the given $\beta$ or Lorentz $\gamma$. We repeat the calculation for various values of $\gamma$ and $\chi$ to obtain a table of values.

The present calculations differ from previous work by Tsuruta \& Takahara (1982) and Melia (1994) in that we integrate over all observer angles whereas those authors restricted themselves to a particular observer angle.

\section{Results}

\subsection{Transition from Cyclotron to Synchrotron.}

Figure 2 shows plots of the emission as a function of scaled frequency $\chi$ for various particle velocities, after the angular integrations in Eq. (22) have been performed. For 
comparison, the dashed lines show the synchrotron formula as given in Eq. (6). We see that the exact numerical results deviate considerably from the limiting synchrotron formula in panels (a)-(c), where the harmonic cyclotron-like emission is quite evident. The harmonics are broadened, partly because of our broadening function $f(\chi)$ (Eq. (21), and partly due to Doppler broadening induced by $\beta_{\|}$(see Eq. (18)). The former effect dominates in panel (a), but the latter is more important in panel (c).

By panel (d), which corresponds to $\beta=0.9, \gamma=2.3$, the harmonics have merged completely at higher values of $\chi$ and the synchrotron approximation is becoming quite good. Yet higher values of $\gamma$ (panels (e)-(f)) make this trend more apparent. However, at low values of $\chi$ the harmonics continue to be present and there are deviations from the synchrotron formula.

Figs. 3a-d show polar plots of the emission as a function of the observer angle $\theta$. In these figures, the particle has $\gamma=10.3$ and is moving in a helix at an angle $\theta_{p}$ to the magnetic field with $\cos \theta_{p}=0.3$. The field points along the positive y-axis. Fig. $3 \mathrm{~d}$ shows the emission pattern that is observed at a large frequency $\chi=100$. According to Fig. 2f, which corresponds to this value of $\gamma$, the emission at this $\chi$ is practically equal to the synchrotron formula. The reason is clear from Fig. 3d. We see that the emission is tightly beamed along the particle direction, and the beam has a half angle $\sim 1 / \gamma$, exactly as assumed when deriving the synchrotron formula. Varying $\cos \theta_{p}$ does not change this behavior and, therefore, on integrating over $\cos \theta_{p}$ we find a total emission which agrees very well with the synchrotron formula.

In strong contrast is the case shown in Fig. 3a, which corresponds to the same values of $\gamma=10.3$ and $\cos \theta_{p}=0.3$, but has a much lower value of $\chi=0.8$. The emission pattern now is not strongly beamed about the particle's velocity. Instead we can clearly see a set of harmonics, each with a width determined by $f(\chi)$. As $\cos \theta_{p}$ varies, the individual beams get longer or shorter, but the harmonic character remains. Therefore, the integrated emission over all $\cos \theta_{p}$ deviates significantly from the synchrotron approximation (as Fig. 2f shows at $\log (\chi) \simeq-0.1$ ). Figs. 3b, c, show how the transition in the beaming occurs as $\chi$ increases.

\subsection{Emission by a Thermal Distribution of Electrons}

The primary output from our calculations is a table of $\eta_{\chi}$ values as a function of electron velocity $\beta$ (or equivalently $\gamma$ ) and dimensionless frequency $\chi$. This table can be convolved with any isotropic velocity distribution to calculate the corresponding spectrum. In the following we describe the results for a thermal Maxwellian distribution. 


\subsubsection{The Ultra-relativistic Regime}

We first consider the ultra-relativistic regime, where $k T \gg m_{e} c^{2}$. Pacholczyk (1970) derived the formulas given by Eqs. (11) and (16), for the emission from a

relativistic Maxwellian distribution of electrons with a fixed particle angle $\theta_{p}$. For an isotropic distribution of particles, we define a new function $I^{\prime}\left(x_{M}\right)$

$$
I^{\prime}\left(x_{M}\right)=\frac{1}{4 \pi} \int I\left(\frac{x_{M}}{\sin \theta_{p}}\right) d \Omega_{p} .
$$

and substitute $I^{\prime}\left(x_{M}\right)$ for $I\left(x_{M}\right)$ in Eq. (11).

Pacholczyk (1970) has numerically calculated $I\left(x_{M}\right)$ over a wide range of $x_{M}$ and tabulated the values. In addition he has shown that in the limit of small $x_{M}$

$$
I\left(x_{M}\right) \rightarrow \frac{1}{x_{M}} \frac{16 \pi}{9 \sqrt{3}}\left(\frac{x_{M}}{2}\right)^{1 / 3} \simeq 2.5593 x_{M}^{-2 / 3} .
$$

In the opposite limit of $x_{M} \gg 1$, Petrosian (1981) finds

$$
I\left(x_{M}\right) \rightarrow 2.5651 \exp \left(-1.8899 x_{M}^{1 / 3}\right) .
$$

We can combine these two limiting extremes into the following simple fitting function,

$$
I\left(x_{M}\right)=2.5651\left(1+\frac{1.92}{x_{M}^{1 / 3}}+\frac{0.9977}{x_{M}^{2 / 3}}\right) \exp \left(-1.8899 x_{M}^{1 / 3}\right),
$$

where we have optimized the coefficient 1.92 in the middle term so as to minimize the error. This fitting function has a maximum error of $0.39 \%$, which occurs when $x_{M} \approx 63$. Figures $4 \mathrm{a}, \mathrm{b}$, compare the fitting function with the exact numerical values of $I\left(x_{M}\right)$ and show the residuals.

In the case of $I^{\prime}\left(x_{M}\right)$, we show in Appendix A. that as $x_{M} \rightarrow 0$

$$
I^{\prime}\left(x_{M}\right) \rightarrow 2.1532 x_{M}^{-2 / 3},
$$

and as $x_{M} \rightarrow \infty$

$$
I^{\prime}\left(x_{M}\right) \rightarrow 4.0505 x_{M}^{-1 / 6} \exp \left(-1.8899 x_{M}^{1 / 3}\right) .
$$

Once again we combine these two limits to obtain a fitting function:

$$
I^{\prime}\left(x_{M}\right)=\frac{4.0505}{x_{M}^{1 / 6}}\left(1+\frac{0.40}{x_{M}^{1 / 4}}+\frac{0.5316}{x_{M}^{1 / 2}}\right) \exp \left(-1.8899 x_{M}^{1 / 3}\right) .
$$

This function has a maximum error of $2.7 \%$ at $x_{M} \approx 160$. Figs. $4 \mathrm{c}, \mathrm{d}$, compare the fitting function with the numerical values and show the residuals. 


\subsubsection{The Mildly Relativistic Regime}

Using our tabulated values of $\eta_{\chi}$ as a function of $\beta$ and $\chi$, we have computed emission spectra for isotropic thermal distributions of electrons with temperatures in the range, $5 \times 10^{8} \mathrm{~K}<T<3.2 \times 10^{10} \mathrm{~K}$. We see that at the highest temperatures the emission is very similar to $I^{\prime}\left(x_{M}\right)$, equation (32), but there are significant deviations at lower temperatures, especially at small $\chi$. We have obtained a set of fitting functions corresponding to each of the temperatures for which we have calculated the spectrum. Each function is of the form

$$
M\left(x_{M}\right)=\frac{4.0505 \alpha}{x_{M}^{1 / 6}}\left(1+\frac{0.40 \beta}{x_{M}^{1 / 4}}+\frac{0.5316 \gamma}{x_{M}^{1 / 2}}\right) \exp \left(-1.8896 x_{M}^{1 / 3}\right),
$$

where $\alpha, \beta, \gamma$, are all adjustable parameters which we have optimized so as to minimize the square of the deviation of $M\left(x_{M}\right)$ from the numerically calculated results. Table 11 shows the optimized parameters we obtained at the various temperatures. We expect that as $T \rightarrow \infty, \alpha, \beta$, and $\gamma$ should all $\rightarrow 1$, since $M\left(x_{M}\right)$ must approach $I^{\prime}\left(x_{M}\right)$. Indeed we see that this is the case in Table 1. At lower values of $T$, however, the parameters are very different from 1 . This is to a large extent because the fitting function is attempting to fit the harmonic "bumps" in the spectrum

In terms of the fitting function $M\left(x_{M}\right)$, the optically thin cyclo-synchrotron emission from a thermal plasma at temperature $T$ is given by (cf. equation (11)),

$$
\varepsilon_{\omega} d \omega=C R(\chi, T) d \omega \quad \operatorname{ergs~s}^{-1} \mathrm{~Hz}^{-1},
$$

with

$$
R(\chi, T)=\frac{\chi M\left(x_{M}\right)}{K_{2}\left(1 / \theta_{e}\right)}
$$

and the power is given by

$$
\omega \varepsilon_{\omega}=\chi L_{\chi}=\omega_{b} C \chi R(\chi, T) \quad \operatorname{ergs~s}^{-1} .
$$

For temperatures $T>3 \times 10^{10} \mathrm{~K}, M\left(x_{M}\right)$ should be replaced by $I^{\prime}\left(x_{M}\right)$ given in equation (32), which is equivalent to setting the fitting constants $\alpha, \beta$, $\gamma$ equal to unity.

Figs. 5, 6, show the fits at various temperatures along with the residuals, and Table 2 shows where the maximum errors occur, both over the range $\log (\chi)<1$ and for $\log (\chi)>1$. We see that the errors are particularly severe for $\log (\chi)<1$ because of the harmonic oscillations which are impossible to fit in detail with a simple function such as (33). However, in many applications, the synchrotron emission will be self-absorbed, and one would be interested primarily in $\log (\chi)>1$. We see that the errors here are much less severe.

We have compared our detailed numerical results with those of Petrosian (1981), Takahara \& Tsuruta (1982) and Melia (1994). We find good agreement with the former 
two papers. The small differences in our results can be explained by the fact that they only considered a single observer direction whereas we have averaged over all directions. We do, however, find a serious discrepancy with Melia's results. For instance, our calculation shows that for $T=10^{10} \mathrm{~K}$, the spectrum peaks at $\log (\chi) \simeq 2$, whereas the calculation by Melia indicates that the peak is at $\log (\chi) \simeq 1$ and that the emission is insignificant at $\log (\chi) \simeq 2$. In fact, Melia's results seem to indicate that the cutoff frequency for the synchrotron emission is essentially independent of the temperature, whereas it is clear from basic principles that the cutoff must increase rapidly with increasing temperature.

\section{Conclusion}

The detailed calculations described in this paper bridge the gap between the limits of nonrelativistic cyclotron emission and ultrarelativistic synchrotron emission. We have calculated the emission due to an isotropic distribution of charged particles moving in a magnetic field, and have shown how the spectrum changes as a function of the particle Lorentz factor $\gamma$ and the dimensionless frequency $\chi$ (defined in Eqs. 20 and 1). Figure 2 shows some results for selected cases. Included in our calculations are all the details of the harmonic emission. This is important at low values of $\gamma$, and for low frequencies even at high values of $\gamma$. Also, we isotropically average over observer directions relative to the magnetic field.

Having calculated emission spectra for an array of values of $\gamma$, we have integrated the spectra over an isotropic relativistic Maxwellian distribution of particle velocities to calculate the spectrum of cyclo-synchrotron emission due to a thermal plasma in a magnetic field. The results are shown in Figs. 5 and 6 for temperatures ranging from $5 \times 10^{8} \mathrm{~K}$ to $3.2 \times 10^{10} \mathrm{~K}$. We have obtained fitting functions $M\left(x_{M}\right)$ with three fitting constants, $\alpha, \beta, \gamma$ (see Eqs. 33-35 and Table 1), which provide a fairly accurate representation of the numerical results. These fitting functions allow the spectrum to be calculated with reasonable accuracy for any temperature $>5 \times 10^{8} \mathrm{~K}$. The errors decrease with increasing temperature in the manner indicated in Table 2.

The thermal cyclo-synchrotron spectra presented in this paper agree with most previous results, except that our calculations are more complete since the spectra have been integrated over all particle and observer angles. At highly relativistic temperatures, our results agree with those given by Pacholczyk (1970), while at mildly relativistic temperatures and for frequencies $\omega \gg \omega_{b}$, our results agree with those of Petrosian (1981) and Takahara \& Tsuruta (1982). There are minor deviations in the results which can be traced to the fact that the previous authors specified a fixed direction of the observer relative to the field rather than averaging over all directions. 
Our results do, however, differ significantly from the calculations presented by Melia (1994) and we have been unable to understand the reason for the discrepancy.

Finally, we note that the basic output of our calculations is a table of emission spectra for isotropic particles of fixed velocity $\beta$ or Lorentz factor $\gamma$. In this paper we have concentrate on one application of this table, namely the calculation of thermal cyclo-synchrotron spectra from thermal plasmas with Maxwellian velocity distributions. The tabulated results could be convolved with any other isotropic electron distribution function, e.g. a power law distribution, to calculate the corresponding spectrum. Our work thus provides a "ready to use" table for determining the cyclo-synchrotron emission from any astrophysical source with an isotropic particle and magnetic field distribution.

Acknowledgements: This work was supported in part by NSF grants AST 9423209 (to the Center for Astrophysics) and PHY 9407194 (to ITP, University of California, Santa Barbara). RN thanks the ITP for hospitality. 


\section{A. Asymptotic Formulae for $I(x)$ and $I^{\prime}(x)$}

\section{A.1. $I(x)$}

The definition of $I(x)$ is given in Eq. (13):

$$
I(x) \equiv \frac{1}{x} \int_{0}^{\infty} z^{2} \exp (-z) F\left(x / z^{2}\right) d z .
$$

By using the approximation of $F(x)$ given in Eq. (9), Pacholczyk (1970) showed that,

$$
I(x) \rightarrow \frac{1}{x} \frac{16 \pi}{9 \sqrt{3}}\left(\frac{x}{2}\right)^{1 / 3} \simeq 2.5593 x^{-2 / 3}, \quad x \ll 1 .
$$

In the opposite limit of $x \gg 1$, we use the approximation of $F(x)$ given in Eq. (10) to write

$$
I(x) \rightarrow \sqrt{\frac{\pi}{2}} \frac{1}{x} x^{1 / 2} \int_{0}^{\infty} z^{2} \frac{1}{z} \exp \left[-\left(z+x / z^{2}\right)\right] d z .
$$

Employing the method of steepest descent, Petrosian (1981) showed that this integral can be evaluated to give

$$
\begin{aligned}
I(x) & \rightarrow \frac{2 \pi}{6^{1 / 2}} \exp \left[-\left(2^{1 / 3}+2^{-2 / 3}\right) x^{1 / 3}\right], \\
& \rightarrow 2.5651 \exp \left(-1.8899 x^{1 / 3}\right), \quad x \gg 1 .
\end{aligned}
$$

\section{A.2. $I^{\prime}(x)$}

The function $I^{\prime}(x)$ is defined by

$$
I^{\prime}(x)=\frac{1}{4 \pi} \int I\left(\frac{x}{\sin \theta_{p}}\right) d \Omega_{p} .
$$

We first consider the case for $x \ll 1$, where we have $I(x) \rightarrow 2.56 x^{-2 / 3}$. Setting $x \rightarrow x / \sin \theta$, we obtain

$$
I^{\prime}(x) \rightarrow 2 \cdot \frac{2 \pi}{4 \pi} \int_{0}^{1} \frac{2.56}{x^{2 / 3}} \sin ^{2 / 3} \theta d(\cos \theta),
$$

which can be evaluated to give

$$
\begin{aligned}
I^{\prime}(x) & \rightarrow \frac{2.56}{x^{2 / 3}} \frac{\Gamma(1 / 2) \Gamma(1 / 3)}{2 \Gamma(11 / 6)}, \\
& \simeq 2.153 x^{-2 / 3}, \quad x \ll 1 .
\end{aligned}
$$


For $x \gg 1$, we have $I(x) \rightarrow 2.5651 \exp \left(-1.8899 x^{1 / 3}\right)$, and setting $x \rightarrow x / \sin \theta$, we obtain

$$
I^{\prime}(x)=2.5651 \int_{0}^{1} \exp \left(-1.8899 x^{1 / 3} / \sin ^{1 / 3} \theta\right) d(\cos \theta) .
$$

We now use the fact that most of the emission comes from $\theta \simeq \pi / 2$. Setting $\theta=\phi+\pi / 2$ we obtain

$$
I^{\prime}(x) \rightarrow 2.5651 \int_{0}^{1} \exp \left(-1.8899 x^{1 / 3} / \cos ^{1 / 3} \phi\right) d(\sin \phi) .
$$

Setting $\cos \phi=\left(1-y^{2}\right)^{1 / 2}$, and Taylor-expanding up to $y^{2}$, we finally obtain

$$
\begin{aligned}
I^{\prime}(x) & \rightarrow 2.5651 \int_{0}^{1} \exp \left(-1.8899 x^{1 / 3}\right) \exp \left(-1.8899 x^{1 / 3} y^{2} / 6\right) d y \\
& =\frac{2.5651 \exp \left(-1.8899 x^{1 / 3}\right)}{2} \sqrt{\frac{\pi}{1.8899}} \sqrt{\frac{6}{x^{1 / 3}}} \\
& \simeq 4.05047 \frac{1}{x^{1 / 6}} \exp \left(-1.8899 x^{1 / 3}\right), \quad x \gg 1 .
\end{aligned}
$$


Table 1: Optimal values of the parameters for different temperatures.

\begin{tabular}{cccc}
\hline$T(K)$ & \multicolumn{3}{c}{$I^{\prime}(x)$} \\
\hline & $\alpha$ & $\beta$ & $\gamma$ \\
\hline $5 \times 10^{8}$ & 0.0431 & 10.44 & 16.61 \\
$1 \times 10^{9}$ & 1.121 & -10.65 & 9.169 \\
$2 \times 10^{9}$ & 1.180 & -4.008 & 1.559 \\
$4 \times 10^{9}$ & 1.045 & -0.1897 & 0.0595 \\
$8 \times 10^{9}$ & 0.9774 & 1.160 & 0.2641 \\
$1.6 \times 10^{10}$ & 0.9768 & 1.095 & 0.8332 \\
$3.2 \times 10^{10}$ & 0.9788 & 1.021 & 1.031 \\
\hline
\end{tabular}


Table 2: List of errors and where they occur for $\log (\chi)<1$ and for $\log (\chi)>1$, for different temperatures.

\begin{tabular}{ccccc}
\hline$T(K)$ & $\log (\chi)<1$ & $\%$ Error & $\log (\chi)>1$ & $\%$ Error \\
& $\log \left(\chi_{\text {max.error }}\right)$ & & $\log \left(\chi_{\text {max.error }}\right)$ & \\
\hline $5 \times 10^{8}$ & 0.1 & 440 & 2.3 & 85.1 \\
$1 \times 10^{9}$ & 0.05 & 33.6 & 3.3 & 16.6 \\
$2 \times 10^{9}$ & 0.0 & 5.9 & 3.0 & 5.3 \\
$4 \times 10^{9}$ & 0.33 & 6.7 & 2.8 & 2.4 \\
$8 \times 10^{9}$ & 0.33 & 5.3 & 1.9 & 0.9 \\
$1.6 \times 10^{10}$ & 0.0 & 7.0 & 1.4 & 0.56 \\
$3.2 \times 10^{10}$ & 0.0 & 7.4 & 1.6 & 0.55 \\
\hline
\end{tabular}




\section{References}

Anderson, M. C., Keohane, J. W., Rudnick, L., 1995, ApJ, 441, 300

Bekefi, G., 1966, Radiation Processes in Plasmas (New York: John Wiley \& Sons, Inc.)

Carilli, C. L., Perley, R. A., Dreher, J. W., Leahy, J. P., 1991, ApJ, 383, 554

Melia, F., 1994, ApJ, 426, 577

Mészáros, P., Laguna, P., Rees, M. J., 1993, ApJ, 415, 181

Narayan, R., Yi, I., Mahadevan, R., 1995, Nature, 374,623

Oster, L., Phys. Rev., 1960, 119, 1444

Oster, L., Phys. Rev., 1961, 121, 961

Pacholczyk, A. G., 1970, Radio Astrophysics (San Francisco: Freeman)

Paczyński, B., Rhoads, J. E., 1993, ApJ, 418, L5

Petrosian, V., 1981, ApJ, 251, 727

Rosner, H., 1958, Republic Aviation Corporation, Missile Systems Division, Technical Report No. 206-950-3, ASTIA AD 208852

Rybicki, G., Lightman, A., 1979, Radiative Process in Astrophysics (New York: John Wiley \& Sons, Inc.)

Schott, G. A., 1912, Electromagnetic Radiation (London: Cambridge University Press)

Schwinger, J., 1949, Phys. Rev., 75, 1912

Takahara, F., Tsuruta, S., 1982, Progress of Theoretical Physics, Vol. 67, No.2, 485 


\section{Figure Captions}

Figure 1: (a) Cyclotron emission for a particle with $\beta=0.1$. The emission is normalized to the total emission in all harmonics. (b) Synchrotron emission for a particle with $\gamma=10$ from standard synchrotron theory.

Figure 2: Plots of scaled emission $\log \left(l_{\chi}\right)$ against $\log (\chi)$ for (a) $\beta=0.2$, (b) $\beta=0.3$, (c) $\beta=0.6$, (d) $\beta=0.9$, (e) $\gamma=5.3$, (f) $\gamma=10.3$, (g) $\gamma=40.3$, (h) $\gamma=100.3$. The vertical axis is the scaled emission, $l_{\chi}$ after the angular integrations in Eq. (22) have been performed. $l_{\chi} \equiv L_{\chi}\left(c / e^{2} \omega_{b}^{2}\right)=L_{\omega}\left(c / e^{2} \omega_{b}\right)$ (cf. Eq.(22)). The dashed lines show the synchrotron limit given by Eqn. (6).

Figure 3: Polar plot of emission from a particle moving with a Lorentz factor $\gamma=10$ at an angle of $\cos \theta_{p}=0.3$. The magnetic field is oriented along the positive $\mathrm{y}$-axis and $\cos \theta=0$ corresponds to the $\mathrm{x}$-axis. The values of $\chi$ are (a) $\chi=0.8$, (b) $\chi=1.4$, (c) $\chi=5.0$, (d) $\chi=100.0$.

Figure 4: (a) Plot of the analytic and numerical values for $I(x)$ and (b) the corresponding residuals. (c) Plot of the analytic and numerical values for $I^{\prime}(x)$ and (d) the corresponding residuals.

Figure 5: (a) Plots of Eqn. (34) (dashed lines) and the numerical calculation (solid lines) for (a) $T=3.2 \times 10^{10}$, (b) $T=1.6 \times 10^{10}$, (e) $T=8 \times 10^{9}$, (f) $T=4 \times 10^{10}$, and their corresponding percentage errors (c), (d), (g), and (h).

Figure 6: (a) Plots of Eqn. (34) (dotted lines) and the numerical calculation (solid lines) for $T=2 \times 10^{9}$, (b) $T=1 \times 10^{9}$, (e) $T=5 \times 10^{8}$, and their corresponding percentage errors $(\mathrm{c}),(\mathrm{d})$, and $(\mathrm{f})$. 

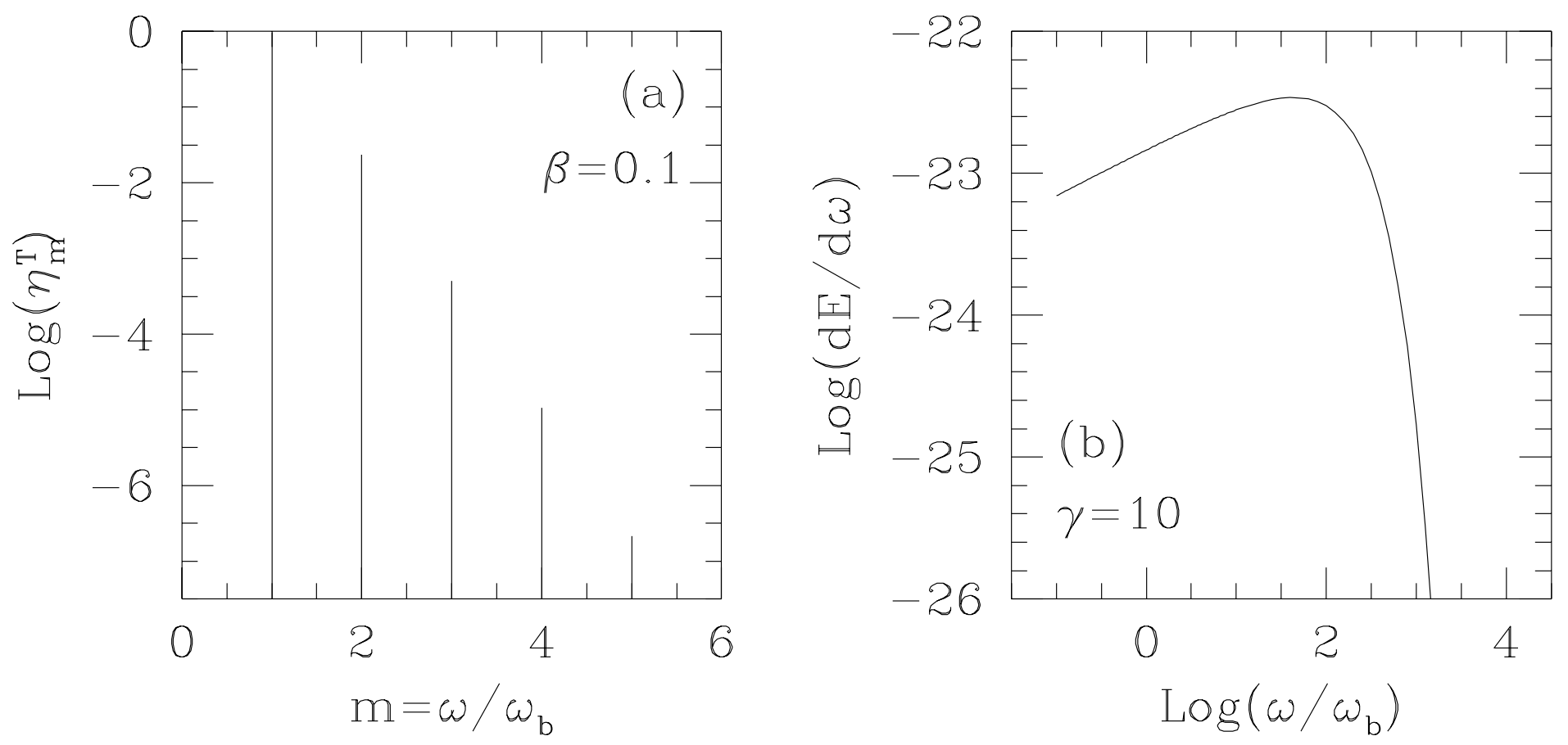

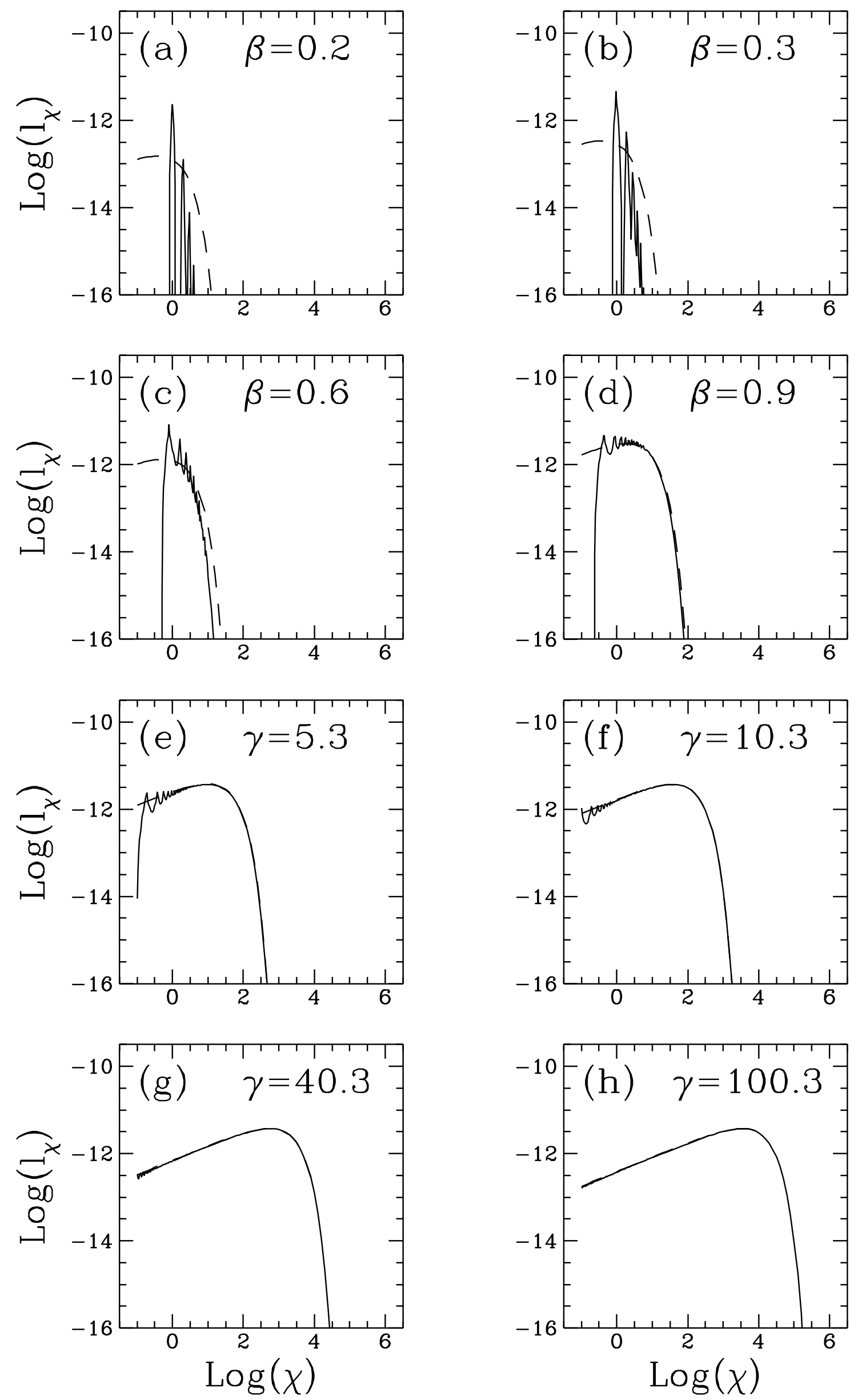

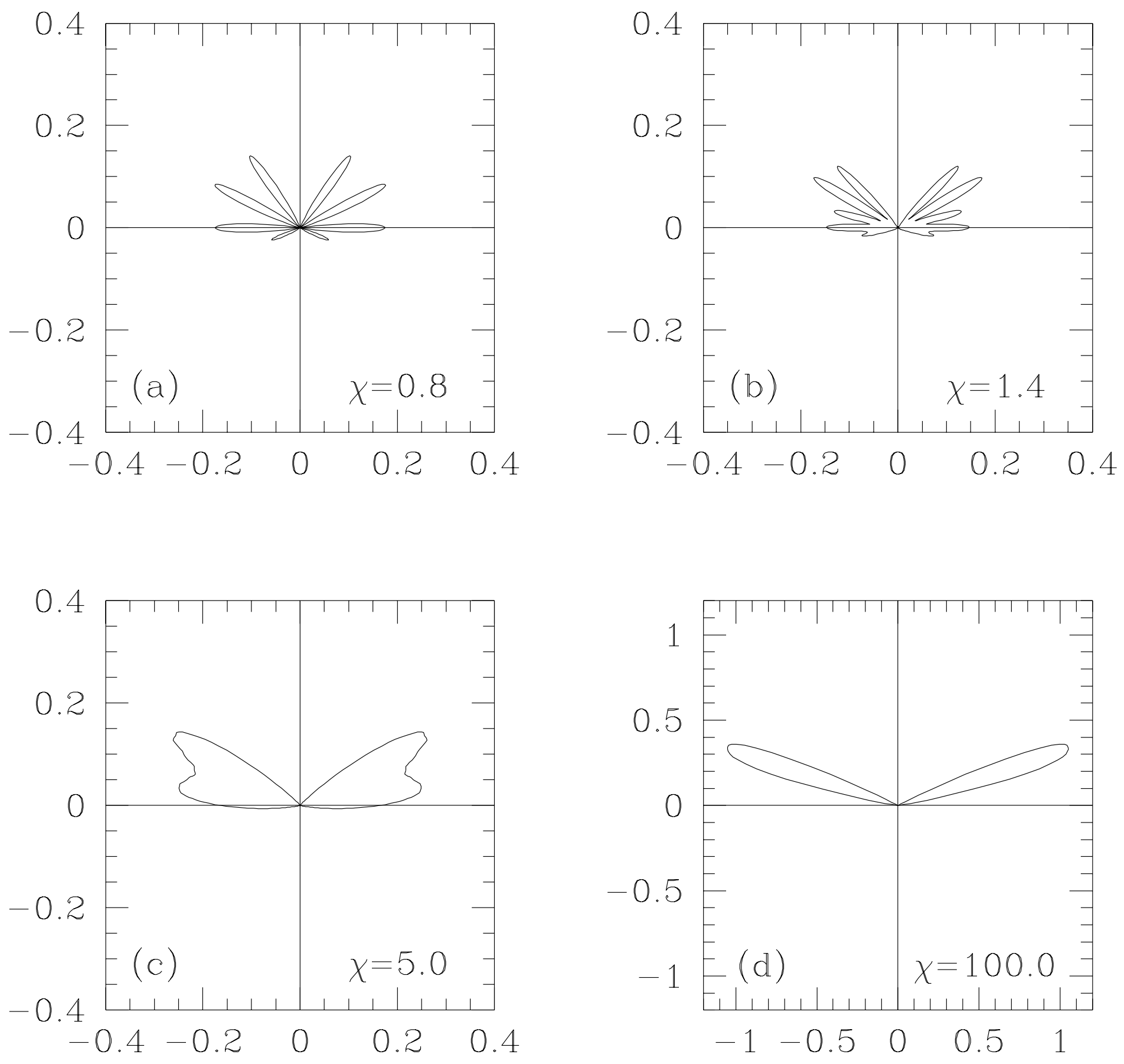

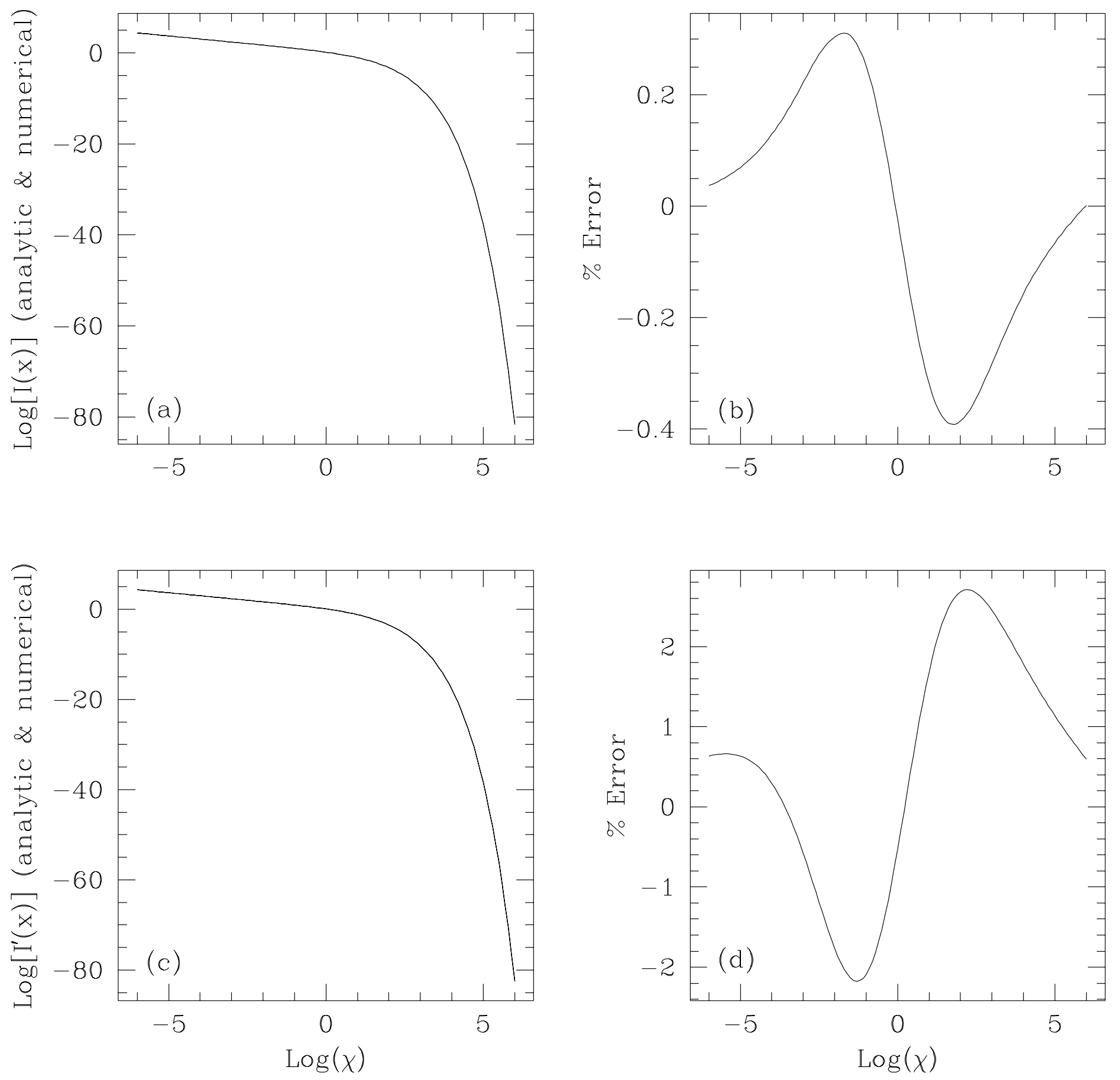

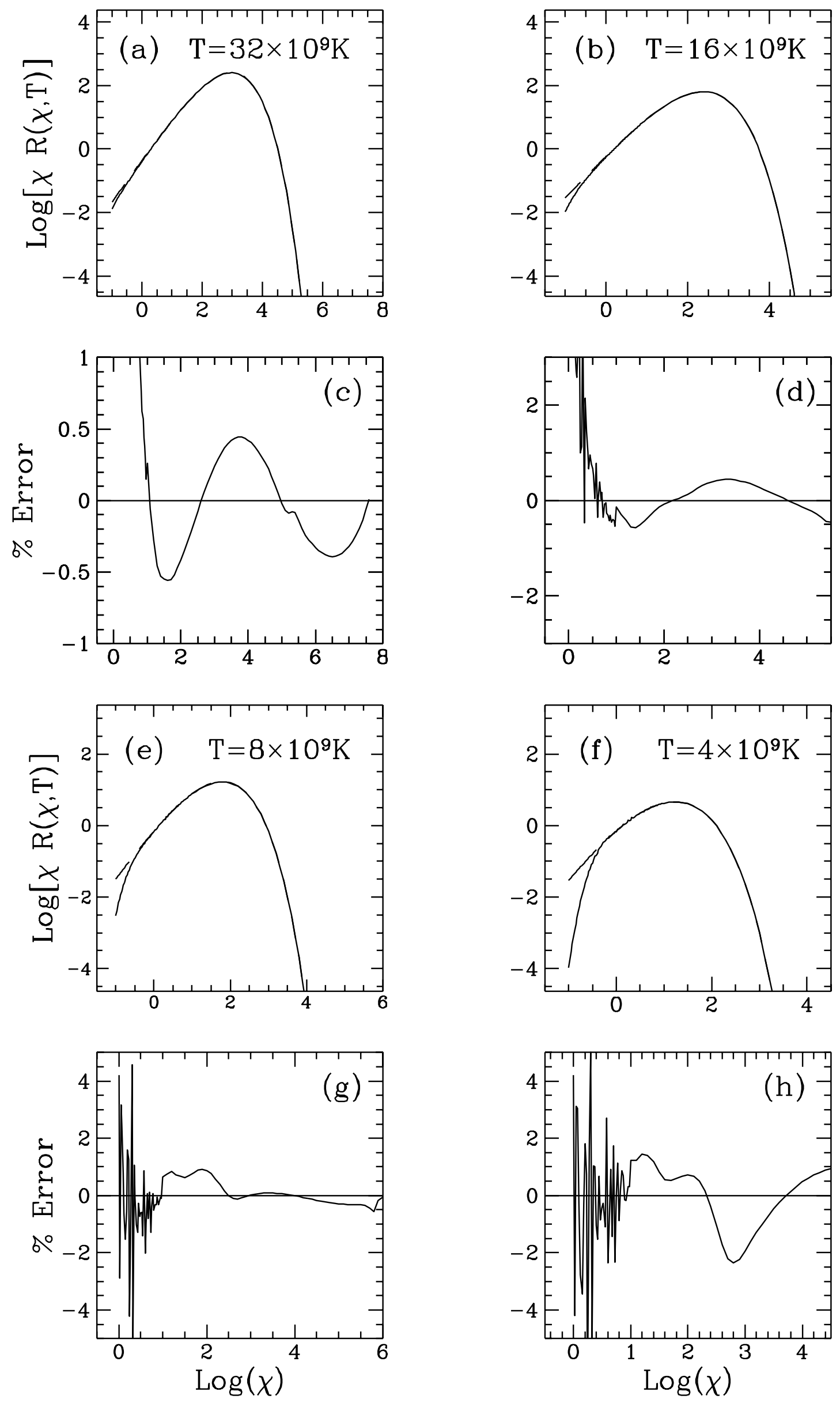

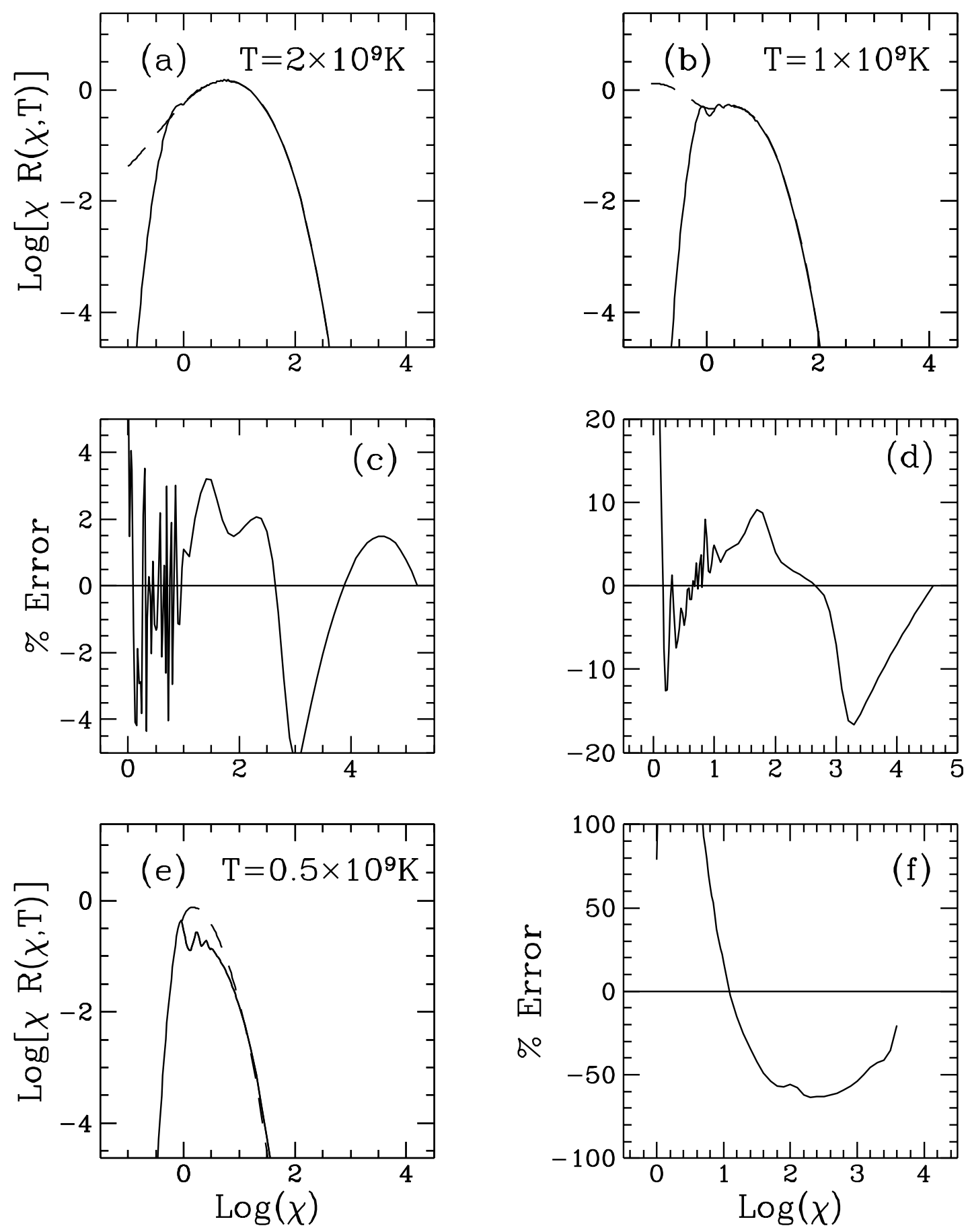